\title{
EFFECT OF SOLVENT BEHAVIOR OF NALIDIXIC ACID BY ULTRAVIOLET SPECTROSCOPY
}

\author{
CARLA MUÑOZ, DANIEL A. PALACIO AND BERNABÉ L. RIVAS * \\ Polymer Department, Faculty of Chemistry, University of Concepción. Casilla 160-C, Concepción, Chile.
}

\begin{abstract}
One of the most widely used pharmaceuticals of the antibiotic type is quinolones. Nalidixic acid belongs to this family of antibiotics and it is widely used in both human and veterinary medicine. The main objective of this work is to show the behavior of nalidixic acid in co-solvent mixtures, in order to study soon the removal of this antibiotic in aqueous solutions or mixtures of water with solvents. The study was carried out by preparing a standard solution containing $25 \mathrm{mg}$ of nalidixic acid in $50 \mathrm{~mL}$ in different solvent mixtures. Subsequently, $20 \mathrm{mg} \mathrm{L}^{-1}$ solutions were prepared using the same mixture of solvents (acetone: water, methanol: water and ethanol: water) to be scanned for wavelengths by UV-Vis spectroscopy. The spectra showed signal displacement in all the mixtures when there was an increase concerning to the water contents due to the interaction of the water with the acid group of the antibiotic. While in mixtures of methanol: water and ethanol: water solutions, there is a variation of the absorption band intensities. As a conclusion, it can be said that it is important to consider the solubility capacity of this antibiotic in different solvents for the purposes of removal studies of this type of emerging contaminants of the antibiotic type.
\end{abstract}

Keywords: Naldixic acid, antibiotic, solvent, ultraviolet spectra.

\section{INTRODUCTION}

Pharmaceutical products and its degradation products have been detected in surface and ground waters in different countries like the United States, Canada, China, and several European countries. These products are introduced into surface waters through different pathways. For example, many consumed pharmaceutical products are not completely absorbed by the body and excreted as waste ${ }^{1}$. These chemicals are unbiodegradable and/or potentially harmful for microbial consortia and environmental systems ${ }^{2}$. They enter into the ecosystems from personal, veterinary, and aquaculture uses, and can persist in the environment and to exhibit deleterious effects in organisms ${ }^{3}$. Among the most important pharmaceutical products are the antibiotics.

One of the more used pharmaceutical antibiotic products are the quinolones. The first quinolone was discovered about 50 years old as an impurity in the chemical manufacture of a batch of the antimalaria agent chloroquine ${ }^{4}$. Nalidixic acid (NA), an antibiotic that belongs to the quinolones is widely used in human and veterinary medicine ${ }^{5}$. The discovery of NA activity in 1962 and its introduction for clinical use as an antimicrobial agent in 1967 can be considered a milestone in the beginning of six decade of development on the quinolone chemistry $^{6}$. It is practically insoluble in water ad its absorption from the gastrointestinal tract was found to be $\mathrm{pH}$-dependent and faster in the non-ionized form ${ }^{7}$. The approach taken to enhance the solubility of a certain drugs depends on the chemical nature of drug substance and the type of drug product desired. In fact, different approaches have been considered before to enhance the nalidixic acid solubility. For example, Maheshwari et al. ${ }^{8}$ utilized hydrotropic solvent as $2.0 \mathrm{M}$ sodium benzoate solution to enhance nalidixic aqueous solubility. El Gindy et al. ${ }^{9}$ improved nalidixic acid solubility by solid dispersion formation with hexamine and piperazine citrate. Co-solvency or the use of solvent mixtures was often considered at early stages and given high priority due to its great solubilization potential and because its safety ${ }^{10}$.

The solubilizing effect achieved by co-solvency can be attributed to the fact that adding an organic co-solvent to water makes the solvating environment less polar, resulting in a more favorable mixing of nalidixic acid in the liquid phase ${ }^{11}$. It is well known that co-solvency power is a function of solvent polarity, which means that solubility should be greater in the least polar solvent ${ }^{12}$. This indicates that the ability of solvent to form hydrogen bonds with the hetero-atoms in the drug molecule is another important factor governing the solubility of drug ${ }^{13}$.

Similar findings have also reported by Seedher and Bhatia, who found the solubility of celecoxib (containing heterocyclic atom) was greater in methanol, despite being more polar than ethanol. This confirms that the polarity of cosolvent is not the only factor governing solubility ${ }^{14,15}$. It is well known the vibrational spectroscopy is a powerful approach to investigate at an atomic level the interaction between organic molecules and their surrounding microenvironment, especially biomacromolecules. The particularity of these techniques is that the spectral contributions arising from the organic molecule can be identified shedding direct light on its interaction ${ }^{16}$.

Molecular vibrations are extremely sensitive to structure and intermolecular interactions (local dielectric constant, presence of $\mathrm{H}$-bond, and presence of nearby charged residues), so that marker band can provide precise information. In particular, when the organic molecule contains carbonyl moieties the effect of intermolecular interactions on the positions of their $\mathrm{C}=\mathrm{O}$ stretching band is generally very strong ${ }^{15,16}$.

Nalidixic acid has one ionizable group at pharmaceutically relevant $\mathrm{pH}$ values, it is the carboxylic acid function at position 3 . The absorbance of a molecule may be affected by its ionization, resulting in a shift in spectrum by changing the $\mathrm{pH}$. The total absorbance of a molecule at any $\mathrm{pH}$ is the sum of the contribution of all the species present ${ }^{17}$.

Spectroscopic techniques are increasingly proposed as alternative analytical methods for the quantification of active pharmaceutical ingredients (APIs) in pharmaceuticals. This due to their advantages over the traditional techniques, such as fast, in-line, non-invasive, and non-destructive measurements without the need of sample preparation ${ }^{19}$.

UV spectroscopy is a widely used quantitative analytical technique that find its application in many research domains and is capable of quantifying very low concentrations $(<0.01 \%)^{18,19}$.

UV spectroscopy has been used to monitor water quality. However, this practice is often limited to a few wavelength measurements. For example, UV 254 has been widely used to monitor hydrophobic organic matter in water and wastewater, used absorbance $285 \mathrm{~nm}$ to measure fulvic acid ${ }^{20}$.

The UV spectrum arises when a sample absorbs electromagnetic radiation of UV range. The ultraviolet region $(200-400 \mathrm{~nm})$ comprises photon energies to excite a molecular electron to a higher energy orbital of many organic substances, i.e. those having lone pair of electrons, double bond, aromatic (conjugated double bond), functional group, such as carboxylic acid, amino, ketone, etc. ${ }^{20}$.

The aim of the current contribution is to show the UV-behavior of the antibiotic nalidixic acid in binary co-solvent mixtures having different hydrogen bonding capacity and different polarity. It is related with the further study to removal of NA water or mixtures of water with other appropriate solvents by using the liquid-phase polymer-based retention, LPR, technique. 


\section{MATERIALS AND METHODS}

\subsection{Preparation of solutions.}

Solutions of nalidixic acid (see Table 1) (98\%, from Sigma-Aldrich, without further purification) was made by dissolving $25 \mathrm{mg}$ of nalidixic acid in $50 \mathrm{~mL}$ of mixed solvent and then were diluted with the same mixed of solvent to obtain 20 $\mathrm{mg} \mathrm{L} \mathrm{L}^{-1}$ of concentration.

The solvent used were bidistilled water, acetone (99\%, Sigma-Aldrich), methanol (99\%, Merck), and ethanol (99\%, Sigma- Aldrich).

The solvent mixtures were the following: acetone-water, methanol-water, and ethanol-water. Different organic solvent- water ratios were prepared as follows: $1: 4,1: 3,1: 2,1: 1,2: 1,3: 1$, and $4: 1$.

Two of these solvents, methanol and ethanol are protic solvents and the other one, acetone is an aprotic solvent. This difference was used to study the influence in the UV-behavior of $\mathrm{H}$-bond, between the nalidixic acid and the different mixtures.

Table 1. Nalidixic acid molecular structure.

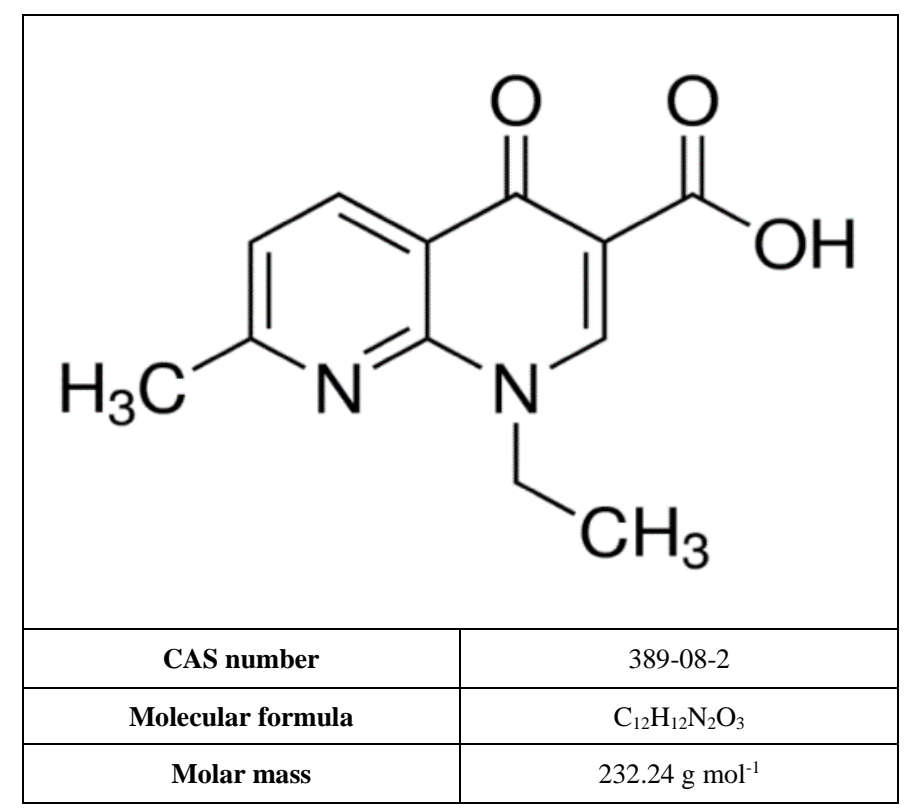

\subsection{UV behavior studies.}

The solvent solutions were analyzed using a UV-visible spectrophotometer (Varian Cary $100 \mathrm{Scan}$ ) between 200 and $500 \mathrm{~nm}$. This spectrophotometer carry out a full wavelength scan in approximately $4.6 \mathrm{~s}$ at $5 \mathrm{~nm}$ data interval and 0.033 sec signal averaging time.

\section{RESULTS AND DISCUSSION}

Nalidixic acid is almost water-insoluble ${ }^{21}$. Its aqueous solubility is reported to be $0.1 \mathrm{mg} \mathrm{L}{ }^{1}$. Its pKa value is 5.95 at $28^{\circ} \mathrm{C}$. Since the solubility of drug in aqueous media determines many aspects of its efficacy for delivery and absorption, different approaches must be first considered to increase nalidixic acid solubility before incorporation into different topical formulations and that is the fundamental scope to the work ${ }^{22}$. UV-Vis spectra to study the solubility of nalidixic acid with an acetone / water mixture, varying the acetone contents (see figure 1) demonstrates that nalidixic acid has two absorption bands at wavelengths of 210 and $340 \mathrm{~nm}$ respectively. However, it can be noted that the variation of the spectrum of the antibiotic at a 1: 1 acetone / water ratio shows an increase in absorbance values due to the low solubility capacity of the solvent mixture towards the antibiotic.

This effect is reflected in an increase in absorbance values, influenced by the lack of a solvent-antibiotic balance. That is, it presents a percentage of undissolved antibiotic solution molecules. On the other hand, as the acetone ratio with respect to the water content increase, the spectra of nalidixic acid are influenced by a widening of the peaks between wavelengths between 315 to 225 $\mathrm{nm}$, but keeping the absorbance approximately constant. This broadening effect may be attributed to low hydrophilicity due to an increased acetone content ${ }^{12}$.

The UV behavior of solutions of nalidixic acid (see figure 1) with different mixtures of solvents were studied and the obtained spectra are shown below:

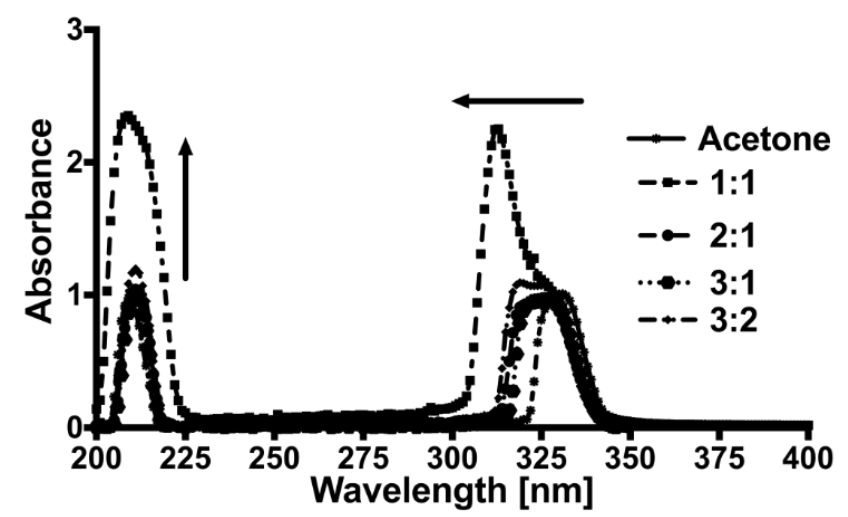

Figure 1. UV absorption spectra of nalidixic acid in different mixed acetone/water. Effect of variation of acetone content

For the studies of the evaluation of the variation of the concentration of water with respect to the contents of acetone in the solution, it can be seen in figure 2 . The spectra of nalidixic acid show slight peak shifts at shorter wavelengths with respect to the corresponding peak at $340 \mathrm{~nm}$ with respect to the pure-antibiotic acetone spectrum. This hypsochromic effect is due to the addition of water. An increase in the absorbance values can also be seen, being 1.5 higher than the mixture with acetone. This increase in absorbance can be attributed to a greater antibiotic-solvent interaction, where the formation of hydrogen bonds intervenes due to an increase in the polarity and hydrophobicity of the compound, which plays a fundamental role in the solubilization capacity of this type of antibiotic.

This increase in absorbance values can be attributed to the greater interactions between the antibiotic molecules and the illumination path (higher solubilization), however, the deformations and instability of the peaks obtained at various contents can be observed using the spectra acetone: water, attributed to the lack of solubility of the antibiotic due to the mixture of solvents. The lack of solubility of this antibiotic may be affected by its solvation capacity and reactivity with polar solvents because it may be susceptible to specific interactions such as hydrogen bonding, protonation, and specific solvation ${ }^{23}$.

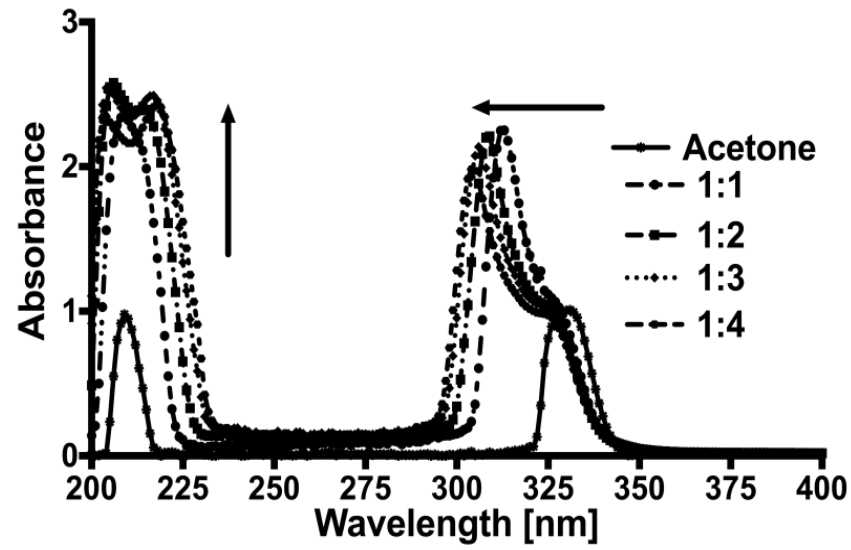

Figure 2. UV absorption of nalidixic acid in different mixtures of acetone/water. Effect of variation of water amount.

When the nalidixic acid is dissolved in pure acetone it can see two peaks at 210 and $340 \mathrm{~nm}$ respectively, and when the quantity of water is increased, the peak at $340 \mathrm{~nm}$ is shifted at the minor wavelength and transformed in two new peaks which is attributed to the hypsochromic effect of the water. This behavior is a consequence of the interaction H-bond between the water and the acid. 


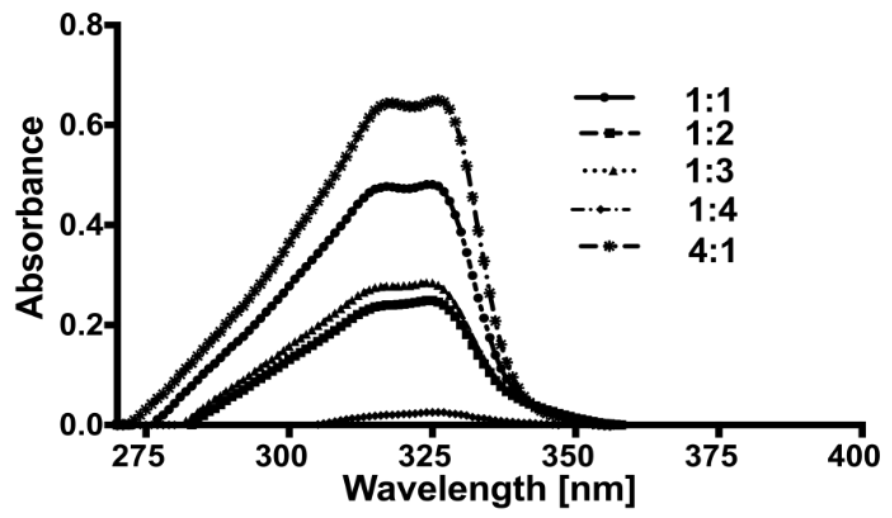

Figure 3. UV absorption of nalidixic acid in different mixed of methanol/water.

In the different mixtures methanol/water it can be observed only one signal around $320 \mathrm{~nm}$, and this peak is maintained, but when the ratio between methanol and water is increased the peak is divided in two peaks. In this case, the H-bond is in all time because the methanol and water have a hydrogen able to generate the H-bond. The spectra in the case of mixtures are similar, but when the ratio of water is increasing the absorption signal is weak.

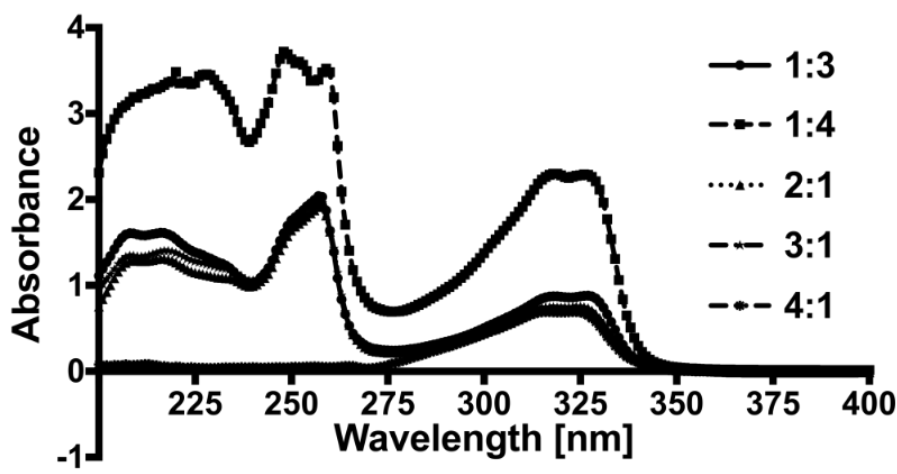

Figure 4. UV absorption of nalidixic acid in different mixed of water/ethanol.

In the different mixtures water/ethanol it can observed several absorption signals and these peaks are maintained in the different mixtures.

The differences between the protic ethanol and methanol mixtures can be due to the distinct polarity of the solvent, because the organic chain in this solvent are different, but both have hydrogen to generated $\mathrm{H}$-bond.

It should be noted that the polarity of the solvent is a function of the solubilization capacity ${ }^{12}$, this means that the solubility presented in practical terms should be greater in the smallest amount of the polar solvent. This characteristic may be due to the presence of the particular solvent and also to their ability to form hydrogen bonds with the heteroatoms of the drug ${ }^{21}$. From this, it can be concluded that the solubility with reference in the case of methanol and ethanol was higher in methanol. Similar results are reported regarding the solubility of the anti-inflammatory drug celecoxib, which contains heteroatoms in its structure, for which a higher solubility in methanol than that in ethanol was found, this solubility being influenced by the aforementioned ${ }^{14,21}$.

\section{CONCLUSIONS}

In all solvent mixtures the increase in the amount of water in the mixture generates a displacement of absorption bands. For the mixtures acetone - water was observed that when the nalidixic acid is studied in pure acetone, an aprotic polar solvent. This showed two absorption bands, which then, due to the addition and increase in the percentage of water are transformed, suffering a displacement and unfolding. This is due to the interaction that water has with the acid, unlike the interaction of acetone, since the water has two hydrogens with which it can generate hydrogen bridges, both with the acid group of the antibiotic, and with the ring of this compound.
In cases, of ethanol/water mixtures, and methanol/water mixture, separately, the absorption signals, regardless of the proportion between two solvents, remain similar in shape, but vary only in intensity, since as is known, these three solvents are polar protic, therefore, their interaction with nalidixic acid should be similar. The notable difference between methanol and ethanol is its polarity, since methanol for having a shorter hydrocarbon chain is more polar and is then more similar to water. This makes its interaction with the different portions of nalidixic acid equal, which is the reason that only an unfolded peak can be observed, approximately at $320 \mathrm{~nm}$ in the spectra of methanol/water mixtures. In contrast, in ethanol/water mixtures, when there is a large proportion of water, the peak can only be observed at approximately $320 \mathrm{~nm}$, but as the ethanol ratio increases several other signals appear below $275 \mathrm{~nm}$, indicating a different interaction between ethanol and acid. These interactions may be due to the organic portion of ethanol. It should therefore be not only considered the solubility of nalidixic acid for further studies of the nalidixic acid in different solvents, but also to take into account its spectrum according to the solvent used, since this will depend on the wavelength chosen for the analyses.

\section{ACKNOWLEDGEMENTS}

The authors are very grateful of FONDECYT (Grant No 1190469) for the financial support.

\section{REFERENCES}

1. Robberson, K. A.; Waghe, A. B.; Sabatini, D. A.; Butler, E. C. Chemosphere 2006, 63, 934

2. Pollice, A.; Laera, G.; Cassano, D.; Diomede, S.; Pinto, A.; Lopez, A.; Mascolo, G. J. Hazard. Mater. 2012, 203-204, 46.

3. Patiño, Y.; Díaz, E.; Ordóñez, S. Chem. Eng. J. 2016, 283, 486.

4. Bisacchi, G. S. J. Med. Chem. 2015, 58, 4874.

5. Wu, Q.; Li, Z.; Hong, H. Appl. Clay Sci. 2013, 74, 66.

6. Bergamini, F. R. G.; Ribeiro, M. A.; Lancellotti, M.; Machado, D.; Miranda, P. C. M. L.; Cuin, A.; Formiga, A. L. B.; Corbi, P. P. J. Mol. Struct. 2016, 1120,115

7. Othman, S.; Muti, H.; Shaheen, O.; Awidi, A.; Al-Turk, W. A. Int. J. Pharm $1988,41,197$

8. Maheshwari, R.; Chaturvedi, S.; Jain, N. Indian J. Pharm. Sci. 2006, 68.

9. Gindy, N. A. E.; Shalaby, A. A.; El-Khalek, H. H. A. Drug Dev. Ind. Pharm. 1983, 9, 363.

10. Chaudhari, P.; Sharma, P.; Barhate, N.; Kulkarni, P.; Mistry, C. Curr. Sci. 2007, 92, 1586.

11. Miyako, Y.; Khalef, N.; Matsuzaki, K.; Pinal, R. Int. J. Pharm. 2010, 393, 48.

12. Miyako, Y.; Zhao, Y.; Takeshima, K.; Kataoka, T.; Handa, T.; Pinal, R. J. Pharm. Sci. 2010, 99, 293.

13. Seedher, N.; Agarwal, P. Indian J. Pharm. Sci. 2009, 71, 82.

14. Seedher, N.; Bhatia, S. AAPS Pharm. Sci.Tech. 2003, 4.

15. Gadalla, M. A. F.; Ghaly, G. M.; Samaha, M. W. Int. J. Pharm. 1987, 38, 71

16. Marrassini, C.; Idrissi, A.; De Waele, I.; Smail, K.; Tchouar, N.; Moreau, M.; Mezzetti, A. J. Mol. Liq. 2015, 205, 2.

17. Ross, D. L.; Riley, C. M. Int. J. Pharm. 1990, 63, 237.

18. Hadad, G. M.; El-Gindy, A.; Mahmoud, W. M. M. Spectrochim. Acta. A. Mol. Biomol. Spectrosc. 2008, 70, 655.

19. Bostijn, N.; Hellings, M.; Van Der Veen, M.; Vervaet, C.; De Beer, T. Anal. Chim. Acta 2018, 1013, 54.

20. Ekanayake, D.; Aryal, R.; Hasan Johir, M. A.; Loganathan, P.; Bush, C.; Kandasamy, J.; Vigneswaran, S. Chemosphere 2019, 233, 245.

21. Tous, S. S.; El Sayed, A. M.; Abd El Mohsen, M. G.; Agban, M. N.; Boushra, M. F. J. Drug Deliv. Sci. Technol. 2012, 22, 341.

22. Grubb, P. E. In Analytical Profiles of Drug Substances; Florey, K., Ed.; Academic Press, 1979; Vol. 8, pp 371.

23. Kolář, P.; Shen, J.-W.; Tsuboi, A.; Ishikawa, T. Fluid Phase Equilibria 2002, 194-197, 771 